\title{
Triple-Junction Solar Cells for Ultra-High Concentrator Applications
}

\author{
Enrique Barrigón, Elisa García-Tabarés, Iván García, Ignacio Rey-Stolle and Carlos Algora \\ Instituto de Energía Solar - Universidad Politécnica de Madrid \\ E.T.S.I. de Telecomunicación ; Avda. Complutense 30 \\ 28040 Madrid (Spain)
}

\begin{abstract}
After the successful implementation of a record performing dual-junction solar cell at ultra high concentration, in this paper we present the transition to a triple-junction device. The semiconductor structure of the solar cells is presented and the main changes in respect to a dual-junction design are briefly discussed. Cross-sectional TEM analysis of samples confirms that the quality of the triple-junction structures grown by MOVPE is good, revealing no trace of antiphase disorder, and showing flat, sharp and clear interfaces between the layers. Triple-junction solar cells manufactured on these structures have shown a peak efficiency of $36.2 \%$ at $700 \mathrm{X}$, maintaining the efficiency over $35 \%$ from 300 to 1200 suns. With some changes in the structure and a fine tuning of its processing, efficiencies close to $40 \%$ at 1000 suns are envisaged.
\end{abstract}

Keywords- III-V semiconductors; photovoltaics; concentrator solar cells, multijunction solar cell; MOVPE

\section{INTRODUCTION}

The main hurdle for the massive penetration of photovoltaic (PV) solar energy into the electricity market has been the high cost of the materials needed in solar cell manufacturing, namely highly pure crystalline semiconductors. Thus, a promising way to reduce the cost of PV electricity is the use of highly efficient solar cells at high concentrations [1]. In terms of cell efficiency, great progress has been made worldwide in the last years with record performing triple-junction solar cells based on III-V semiconductors with efficiencies close to $42 \%$ [2]. However, these excellent peak efficiencies where always obtained at concentrations ranging from 150 to 400 suns. These levels are still low to counterbalance the elevated cost of the substrates and processing techniques needed for high concentration as compared to conventional crystalline silicon PV or new thin film technologies. Our cost calculations show that the most advantageous concentration range to exploit such devices is ultra high concentration (UHCPV), namely at irradiances beyond 1000 suns. At this illumination, costs below $1 € / \mathrm{Wp}$ can be achieved for the whole PV plant assuming small solar cells (in the range of square millimeters) that take full advantage of mature optoelectronic processing techniques and fast learning curves [1]. After the successful implementation of a record performing dual-junction solar cell at high concentration [3], in this paper we present the optimization of key aspects in the transition to a triple-junction device and the results of the triple-junction solar cells manufactured.

\section{TRIPle JunCtion Solar Cell Design For UHCPV}

\section{A. From Dual-junction to Triple Junction}

Recently, our group presented a record-performing GaInP/GaAs dual-junction solar cell with a maximum efficiency of $32.6 \%$, which remained virtually constant for concentrations ranging from 400 to 1000 suns [3]. Moreover, the efficiency didn't decrease steeply above 1000 suns but stayed above $30 \%$ up to irradiances above 3500 suns [3]. This device demonstrated that the attainment of high efficiencies at 1000 suns and above is a reachable and realistic target. However, despite this excellent result it is clear that a dualjunction solar cell -though having interesting applications in approaches that aim substrate reuse, need substrate removal [4] or spectral splitting- has a limited efficiency potential when compared to state-of-the-art triple-junction solar cells. Accordingly, this methodology for UHCPV design is being applied to the development of lattice matched GaInP/GaInAs/Ge triple-junction devices, with the goal of achieving efficiencies in excess of $40 \%$ at 1000 suns and above.

The transition from a dual-junction solar cell based on GaAs to a triple-junction device grown on germanium is not a straight forward task. In fact, the evolution to a triple-junction solar cell forces key changes in the device. The main modification is of course related to having to deal with a new substrate $-\mathrm{a}$ germanium wafer- which will act both as the mechanical support for the whole device as well as the third subcell. In addition, there are more subtle changes such as: (a) the need to modify the composition of virtually all the layers in the middle and top subcells to cope with the slight difference in lattice constant between Ge and GaAs; and (b) a second tunnel junction has to be added between the bottom and middle subcell, which has to be fine tuned to withstand the thermal load associated to the growth of the middle and top subcell without degradation of the tunneling characteristics. All these changes configure a complicated semiconductor structure that can be seen in figure 1.a. This structure consists of around 30 layers, involving six different materials, using four different dopants. A more detailed discussion of these changes, including the optimization of the heteronucleation process on germanium can be found elsewhere [5-6]. 


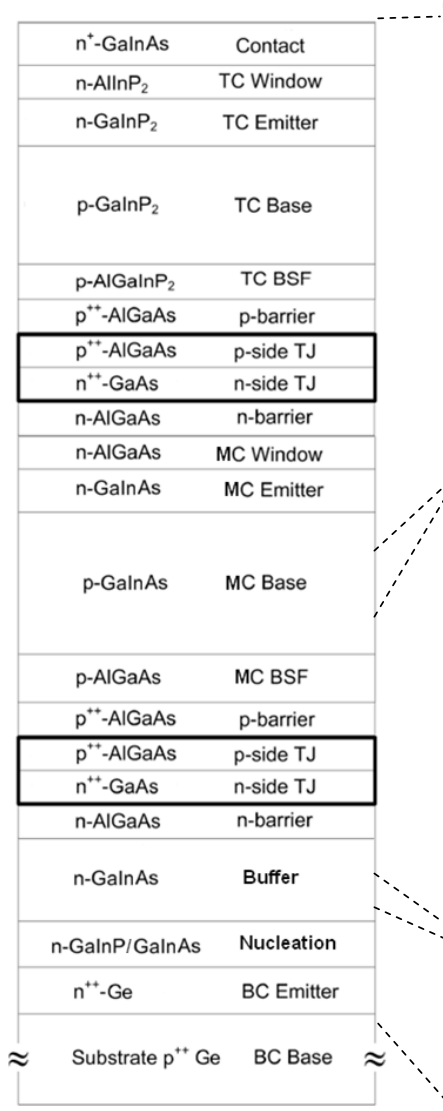

(a)

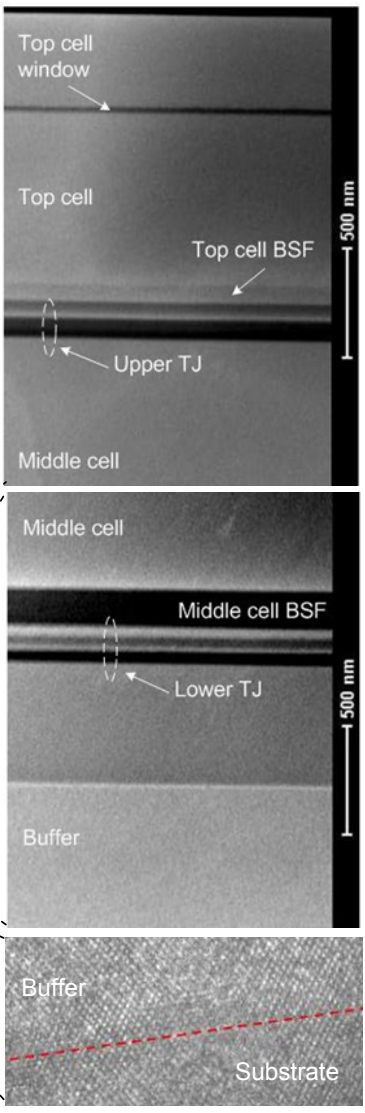

(b)
Figure 1. (a) Target triple-junction solar cell semiconductor structure. (b) TEM analysis of the structure manufactured

\section{B. Structure growth and characterization}

Triple-junction solar cells with the structure depicted in figure 1.a were grown by low-pressure metalorganic vaporphase epitaxy (MOVPE) in a horizontal reactor. Substrates used were (100) Ge with a miscut angle of $6^{\circ}$ towards the nearest (111) plane. The precursors used were arsine and phosphine for the group- $\mathrm{V}$ elements, and trimethyl gallium, trimethyl aluminum, trimethyl indium, ditertiarybutyl silane, carbon tetrabromide, diethyl telluride, and dimethyl zinc for the group-III and dopant elements. Carrier gas was Pd-purified hydrogen, the pressure of the process was 100 mbar and temperatures used ranged from $550^{\circ} \mathrm{C}$ to $675^{\circ} \mathrm{C}$.

In order to confirm the quality of the structures, cross sections of these samples were prepared and analyzed by high resolution TEM and STEM (figure 1.b). The lower part of figure 1.b shows a HRTEM image of the III/V-germanium heterointerface. This image evidences that the epilayer/ substrate interface is virtually indistinguishable (a red dotted line has been included as a guide-to-the-eye). No trace of antiphase domains or boundaries were found in any of the HRTEM images taken at this interface, confirming the suitability of the nucleation procedure developed. In the upper images in figure 1.b, STEM images of the lower and upper parts of the triple-junction solar cell structure are also shown. These figures show no trace of defects, with flat, sharp and clear interfaces, confirming the quality of the MOVPE process.

\section{Device Results}

\section{A. Device manufacturing}

The triple-junction solar cell structures discussed in the previous section were processed into high concentrator devices following a procedure very similar to that developed for our record dual-junction solar cell [3]. Photolithography techniques were employed to define the front grid, with inverted square symmetry and a shadowing factor of around $4 \%$. The front $(\mathrm{AuGe} / \mathrm{Ni} / \mathrm{Au})$ and back $(\mathrm{Au})$ metal contacts were deposited by means of thermal evaporation. The wet mesa etching process applied to isolate the devices and minimize the perimeter recombination consisted of an optimized sequence of etching processes using acid and basic solutions. A double-layer antireflecting coating (ARC) formed by $\mathrm{MgF}_{2} / \mathrm{ZnS}$ was finally deposited on the devices, which were then encapsulated.

\section{B. External quantum efficiency}

Figure 2 summarizes the external quantum efficiency (EQE) of the triple junction solar cells manufactured (EQE of Ge subcell not plotted for clarity). The EQE of the record dualjunction solar cell is also included for comparison. As shown in the figure, both curves virtually overlap (except at the bandgap edges due to differences in composition) indicating that the quality of minority carrier properties is very similar in both devices. The fitting of these curves to conventional analytical models suggests that the response in the top cell is somewhat low at the short wavelength range $(300-500 \mathrm{~nm})$ presumably due to the high doping of the emitter needed to keep the solar cell series resistance low. On the other hand, the response in the top cell at its high wavelength range $(500-700 \mathrm{~nm})$ is absorption limited, indicating that minority carrier properties in the base are far from limiting the performance of the device. Regarding the (In)GaAs cell, its largest limitation is the loss of photocarriers absorbed in the GaAs cathode of the upper tunnel junction. The overall result is that the device is middle cell limited with a short circuit current of $\mathrm{J}_{\mathrm{SC}}=13.3 \mathrm{~mA} / \mathrm{cm}^{2}$ (AM1.5D ASTM G173 normalized to $1000 \mathrm{~W} / \mathrm{m}^{2}$ ), being the current in the top cell $\mathrm{J}_{\mathrm{SC}, \mathrm{TC}}=14.2 \mathrm{~mA} / \mathrm{cm}^{2}$. This situation reveals a relatively large current mismatch of around $6.5 \%$ than can be easily corrected adjusting the thickness of the top and middle subcells.

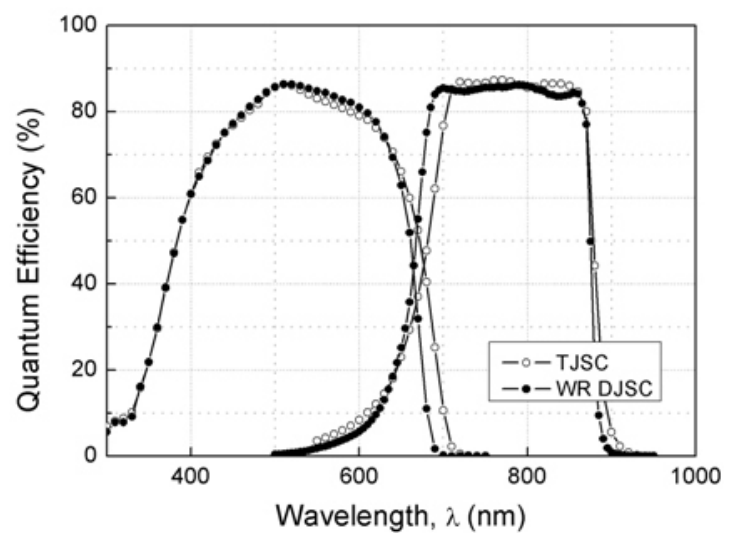

Figure 2. EQE of the triple-junction solar cells (TJSC) developed. The world record dual-junction solar cell (WR DJSC) is included for comparison. The $\mathrm{EQE}$ of the Ge subcell in the TJSC is not shown for clarity 


\section{Concentrator results}

Figure 3 presents the concentrator results of the triplejunction solar cells developed. Again, the analogous results for the dual-junction world-record device are included for comparison. As this figure shows, for the triple-junction solar cell efficiency is over $36 \%$ for concentrations between 550 and 850 suns, peaking at $700 \mathrm{X}$ with a $36.2 \%$. After this maximum, the drop in efficiency is much steeper than in the case of the record dual-junction solar cell as a result of the rapid decrease of the fill factor after 500X. Preliminary investigations indicate that this behavior is related to an incidental processing issue, which caused an increase in the series resistance of the device (excessive underetching of the contact layer that caused an increase in the front contact series resistance). It is important to note that the new series resistance components associated to the triple-junction structure (i.e. the germanium wafer and the additional tunnel junction) should only add minor contributions to the overall series resistance as compared to the dual-junction design and thus the fill factor evolution versus concentration in both designs should be similar. Finally, the open circuit voltage in the triple junction device is around $310 \mathrm{mV}$ higher than in the dual-junction at $1000 \mathrm{X}$ once again confirming a successful integration of the germanium subcell in the device and a lack of a significant amount of threading dislocations in the structure.
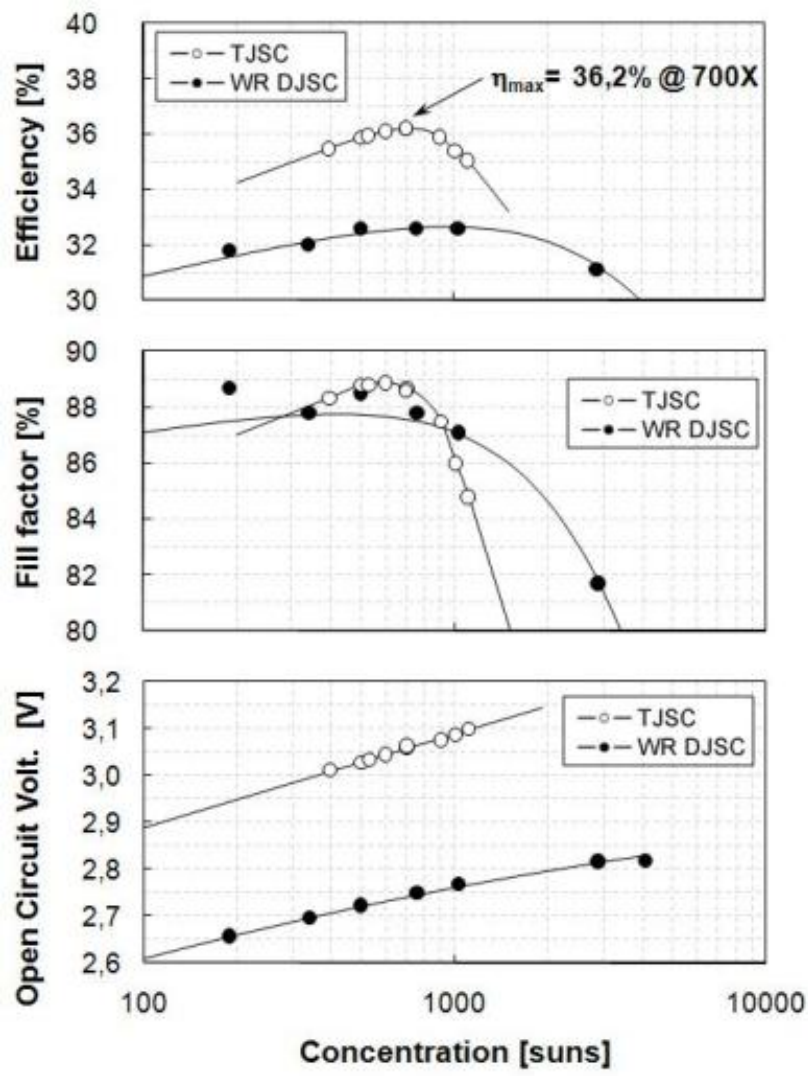

Figure 3. Concentration response of triple-junction solar cells (white circles). The results or the world record dual-junction solar cell (black circles) is also plotted for comparison.

\section{Improving the results}

The device analysis performed suggests evident changes to improve the efficiency of these solar cells. Firstly, to recover the fill factor, processing issues have to be solved and the series resistance of the device has to be kept to its design value. Photocurrent can be increased by simply readjusting the thickness of both subcells until current matching is reached. However, further boosts in short circuit current can be reached changing the design of the tunnel junction. As shown in figure 1.a, the top tunnel junction used in the current version of the triple-junction solar cell is based on a $\mathrm{p}^{++} \mathrm{AlGaAs} / \mathrm{n}^{++} \mathrm{GaAs}$ heterostructure doped with carbon and tellurium. The use of the appropriate growth conditions led to the attainment of an unparalleled electrical performance in these tunnel junctions. In fact, as shown in figure 4 , a peak current density as high as $10,1 \mathrm{kA} / \mathrm{cm}^{2}$ and a series resistance at $0 \mathrm{~V}$ bias as low as $1,6 \cdot 10^{-5} \Omega \cdot \mathrm{cm}^{2}$ were obtained. These electrical characteristics are far better than necessary for the attainment of an excellent performance, concerning series resistance, for any light concentration achievable on Earth.

However, the lower optical transmission exhibited by low band gap tunnel junctions, as compared to higher band gap approaches, gives rise to a lower $\mathrm{J}_{\mathrm{SC}}$ in the middle subcell. Thereby, the use of high band gap tunnel junctions, typically based on $\mathrm{p}^{++} \mathrm{AlGaAs} / \mathrm{n}^{++} \mathrm{GaInP}$ heterostructures, allows the achievement of higher photocurrents in the solar cell, but, in general, leads to worse electrical properties and a higher complexity in their development, concerning the MOVPE growth of their semiconductor structure. We have carried out some preliminary experiments growing high band gap tunnel junctions based on the $\mathrm{p}^{++} \mathrm{AlGaAs} / \mathrm{n}^{++} \mathrm{GaInP}$ heterojunction. The results obtained so far exhibit a series resistance more than one order of magnitude higher than in the case of the $\mathrm{p}^{++} \mathrm{AlGaAs} / \mathrm{n}^{++} \mathrm{GaAs}$ tunnel junction, as can be observed in Figure 4. Nevertheless, and bearing in mind that the current density in these devices at 1000 suns is around $14,5 \mathrm{~A} / \mathrm{cm}^{2}$, this performance is enough to obtain a high efficiency solar cell.

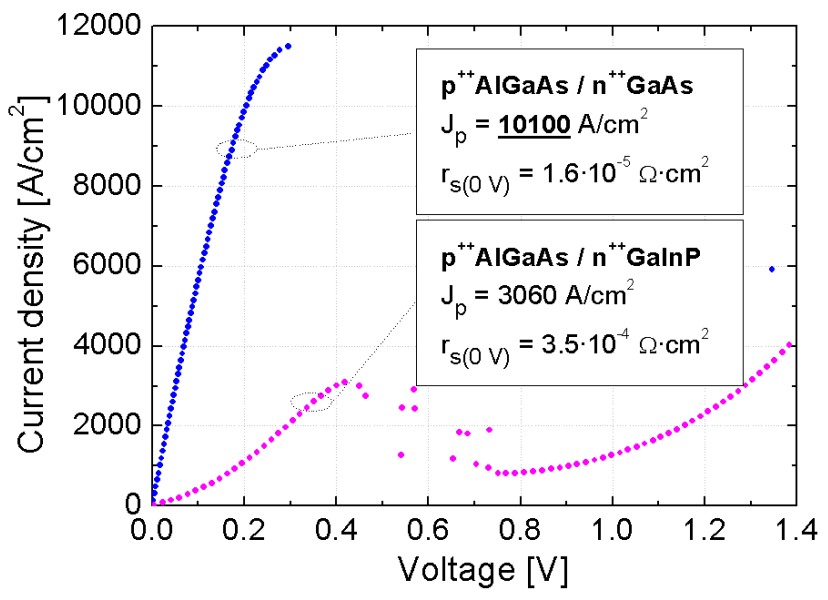

Figure 4. J-V curve of high performance $\mathrm{p}^{++} \mathrm{AlGaAs} / \mathrm{n}^{++} \mathrm{GaAs}$ and $\mathrm{p}^{++} \mathrm{AlGaAs} / \mathrm{n}^{++} \mathrm{GaInP}$ tunnel junctions 
An additional push in efficiency can be obtained increasing the open circuit voltage of the device. To do so, the bandgap of the top cell has to be increased by disordering the GaInP. The use of surfactant mediated growth (particularly adding some $\mathrm{Sb}$ during the growth of the top cell [7]) can raise the top cell bandgap up to $1,85 \mathrm{eV}$. This increase should bring the $\mathrm{V}_{\mathrm{OC}}$ at $1000 \mathrm{X}$ around $3,150 \mathrm{~V}$.

The combination of these three improvements at 1000 suns -i.e. a short circuit current of $14,5 \mathrm{~A} / \mathrm{cm}^{2}$; a $\mathrm{V}_{\mathrm{OC}}$ of $3,15 \mathrm{~V}$ and a fill factor of $88 \%$ as in the dual-junction record deviceshould bring efficiency above $40 \%$.

\section{SUMMARY AND CONCLUSIONS}

Ultra High Concentrator PV ( $>1000$ suns) based on III-V multi-junction solar cells seems a robust strategy to decrease the cost of PV electricity. The design of high efficiency devices at such high concentrations presents multiple challenges. After the successful implementation of a record performing dualjunction solar cell at ultra high concentration, in this paper we have presented the transition to a triple-junction device. TEM analysis confirms that the quality of the triple-junction structures grown by MOVPE is good, revealing no trace of APDs, and showing flat, sharp and clear interfaces. Triplejunction solar cells manufactured on these structures have shown a peak efficiency of $36.2 \%$ at $700 \mathrm{X}$, with a value over $35 \%$ from 300 to 1200 suns. Simulations indicate that by implementing a high bandgap top cell and top tunnel junction, efficiencies of $40 \%$ at $1000 \mathrm{X}$ are reachable

\section{ACKNOWLEDGMENTS}

We thank J. Bautista for assistance in the maintenance of the MOVPE reactor and the solar cell processing line. The TEM work was carried out at the LABMET of the Universidad Carlos III de Madrid.
This work was supported by the Spanish Ministerio de Ciencia e Innovación under the CONSOLIDER-INGENIO 2010 program by means of the GENESIS FV project (CSD2006-004) and the research projects with references TEC2009-11143, TEC2008-01226 and PSS-440000-2009-30. The Comunidad de Madrid has also contributed under the NUMANCIA II Programme (S2009/ENE1477).

\section{REFERENCES}

[1] C. Algora, I. Rey-Stolle, I. García, B. Galiana, M. Baudrit, P. Espinet, E. Barrigón and J. R González, "III-V multijunction solar cells for ultrahigh concentration photovoltaics", 34th IEEE PVSC, Philadelphia (USA), May 2009

[2] R. R. King, A. Boca, W. Hong, X.-Q. Liu, D. Bhusari, D. Larrabee, K. M. Edmondson, D. C. Law, C. M. Fetzer, S. Mesropian, and N. H. Karam, "Band-gap-engineered architectures for high-efficiency multijunction concentrator solar cells", 24th European Photovoltaic Solar Energy Conference, Hamburg (Germany), September 2009

[3] I. García, I. Rey-Stolle, B. Galiana, and C. Algora, “A 32.6\% efficient lattice-matched dual-junction solar cell working at 1000 suns", Appl. Phys. Lett. 94, 053509 (2009)

[4] J. Yoon, S. Jo, I. S. Chun, I. Jung, H-S Kim, M. Meitl, E. Menard, X. Li, J. J. Coleman, U. Paik, J. A. Rogers, "GaAs Photovoltaics and Optoelectronics Using Releasable Multilayer Epitaxial Assemblies" Nature, Vol. 465, pp. 329-333 (2010)

[5] I. Rey-Stolle, C. Algora, E. Barrigón, E. García-Tabarés, I. García, P. Espinet, "Status of ultra-high concentrator multijunction solar cell development at IES-UPM", 25th European Photovoltaic Solar Energy Conference, Valencia (Spain), September 2010

[6] E. Barrigon, B. Galiana, I. Rey-Stolle, "Reflectance anisotropy spectroscopy assessment of the MOVPE nucleation of GaInP on germanium (1 $\left.\begin{array}{lll}1 & 0\end{array}\right)$ ", Journal of Crystal Growth (in press), available online 24 September 2010

[7] J.M. Olson, W.E. McMahon, and S. Kurtz, "Effect of Sb on the Properties of GaInP Top Cells", 4th World Conference on Photovoltaic Energy Conversion (WCPEC-4), Hawaii (USA), May 7-12, 2006 